\title{
Caractérisation moléculaire d'isolats du virus de la maladie de Gumboro provenant d'élevages avicoles semi-intensifs de la région de Dakar au Sénégal
}

\author{
Alkaly BADJI ${ }^{1 *}$, Modou MOUSTAPHA LO ${ }^{2}$ et Rianatou BADA ALAMBEDJI ${ }^{3}$ \\ ${ }^{1}$ Université de Thiès, Institut Supérieur de Formation Agricole et Rurale, BP 54 Bambey, Sénégal. \\ ${ }^{2} I S R A / L N E R V$, Laboratoire National de l'Elevage et de Recherches Vétérinaires, BP 2057 Dakar-Hann, \\ Sénégal. \\ ${ }^{3}$ EISMV, Ecole Inter-Etats des Sciences et Médecine Vétérinaires, BP 5027 Dakar-Fann, Sénégal. \\ *Auteur correspondant ; E-mail : badjialkaly@yahoo.fr ; Tel : +221 775665609
}

\section{RESUME}

Au Sénégal, des foyers de la maladie de Gumboro sont souvent observés dans les élevages où elle provoque des pertes économiques importantes. Une étude portant sur cette maladie dans les élevages avicoles semi-intensifs de la région de Dakar a été conduite. Elle a consisté à identifier sur le plan moléculaire les souches circulant et à étudier les liens de parenté génétique qui existent entre les souches sénégalaises et celles étrangères en vue de mieux réadapter les stratégies de lutte. Pour cela, la région hypervariable du génome viral codant pour le « domaine variable de la protéine VP2 » a été séquencée. Les séquences d'acides nucléidiques et aminés comparées à des séquences publiées sur la base de données de GenBank. Ainsi, les résultats indiquent une forte prévalence des souches "hypervirulentes ». Toutes les huit souches étudiées appartiennent au génotype des souches «hypervirulentes ». Parmi ces souches, une est classée dans le sous-génotype VV3 et les autres dans VV2. Les souches de ce sous-génotype sont très proches des souches nigérianes et constituent avec des souches tanzaniennes, zambiennes et éthiopiennes la lignée africaine VV2. Toutefois, des investigations portant sur le segment B permettraient d'apporter plus de précisions sur l'origine des souches qui circulent à Dakar.

(c) 2019 International Formulae Group. All rights reserved.

Mots clés : Caractérisation moléculaire, souches hypervirulentes, Sénégal, Maladie de Gumboro.

\section{Molecular characterization of Gumboro disease virus isolate from semi- intensive poultry farms in Dakar, Senegal}

\begin{abstract}
In Senegal, outbreaks of Gumboro disease are often observed with significant economic losses. A study of this disease in semi-intensive poultry farms was carried in Dakar. The research work consisted to identify by molecular biology the viral strains circulating and study the genetic relationship between Senegalese strains and foreign strains in order to better rehabilitate the control strategies. To do so, the hypervariable region of the viral genome encoding "the VP2 variable domain" was sequenced. The nucleic and amino acid sequences were compared to sequences published on the GenBank data base. Thus, the results indicate a high prevalence of "hypervirulent" strains. All eight strains studied belong to the genotype of "hypervirulent" strains. Among these
\end{abstract}


strains, one strain is classified in sub genotype VV3 and the others in VV2. The strains of this sub genotype are very close to the nigerian strains and constitute with the tanzanian, zambian and ethiopian strains the African sub genotype VV2. However, investigations on segment B would provide more details on the origin of viral strains circulating in Dakar.

(C) 2019 International Formulae Group. All rights reserved.

Keywords: Molecular characterization, hypervirulent strains, Senegal, Gumboro disease.

\section{INTRODUCTION}

La maladie de Gumboro est due à un virus à ARN de la famille des Birnaviridae. Les souches du virus sont classées en deux sérotypes désignés sérotype 1 et 2 (Mac Lachlan et Dubovi, 2011). Le sérotype 1 est pathogène pour la volaille alors que le sérotype 2 est apathogène et a été isolé sur le poulet et la dinde. La maladie affecte les jeunes poulets âgés de 3 à 6 semaines. Le virus a tendance à se propager et à persister dans des milieux où les conditions d'hygiène sont mauvaises. Une implication des rongeurs dans la transmission du virus au sein des exploitations où le fumier est stocké à côté des bâtiments d'élevage a été rapportée au Sénégal (Badji et al., 2017). Une telle pratique est aussi observée au Bénin par Boko et al. (2015).

Plusieurs études menées en Afrique sur le virus (Owoade et al., 2004; Hassan, 2004 ; Mardassi et al., 2004 ; Kasanga et al., 2013 ; Jembery et al., 2014) ont rapportées que les souches virales du sérotype 1 peuvent être classées dans trois génotypes: le génotype des souches virulentes « classiques»,celui des souches «variantes » et celui des souches « hypervirulentes ».

Ces dernières, plus pathogènes que les souches « classiques », sont capables d'infecter des sujets porteurs de taux d'anticorps habituellement protecteurs. Et l'émergence de ces souches a rendu depuis quelques années les perspectives de lutte contre la maladie encore plus complexes.

$\mathrm{Au}$ Sénégal, la maladie est diagnostiquée pour la première fois en 1975 dans des élevages avicoles de la région de Dakar. Dès lors, elle constitue un véritable obstacle au développement de l'aviculture moderne. D'où l'intérêt de connaitre les caractéristiques des souches virales isolées à l'occasion de cas cliniques de la maladie. En effet, à ce jour, seules deux études se sont intéressées à la caractérisation moléculaire des souches virales circulant au Sénégal (Eterradossi et al., 1999 ; Badji et al., 2016 ). Ce qui fait que, des inconnues méritent d'être élucidées sur ce virus.

C'est dans ce contexte que la présente étude a été conduite à partir d'isolats de 2012, 2014 et 2006 provenant d'élevages avicoles semi-intensifs de la région de Dakar. L'objectif de l'étude est d'identifier les souches virales circulantes dans ces élevages et étudier les liens de parenté génétique qui existent entre ces souches et celles isolées en Europe, en Asie et en Afrique en vue de pouvoir mieux réadapter les stratégies de lutte entreprises depuis longtemps.

\section{MATERIEL ET METHODES \\ Cadre d'étude}

L'étude a concerné la région de Dakar qui est située dans la presqu'île du Cap Vert. Elle s'étend sur une superficie de $550 \mathrm{~km}^{2}$, soit $0,28 \%$ du territoire national. Par la présence d'une façade maritime ceinturant presque toute la région, le climat est caractérisé, pendant une bonne période de l'année, par un microclimat très favorable aux activités avicoles. La Région de Dakar abrite plus de $80 \%$ des élevages avicoles semi-intensifs (Traoré, 2006) et concentre toutes les entités de base intervenant dans la filière avicole moderne au Sénégal. Les isolats du virus de la maladie de Gumboro étudiés provenaient des élevages avicoles semi-intensifs des communes de Bambilor, Pout et Thiaroye (Figure 1). 


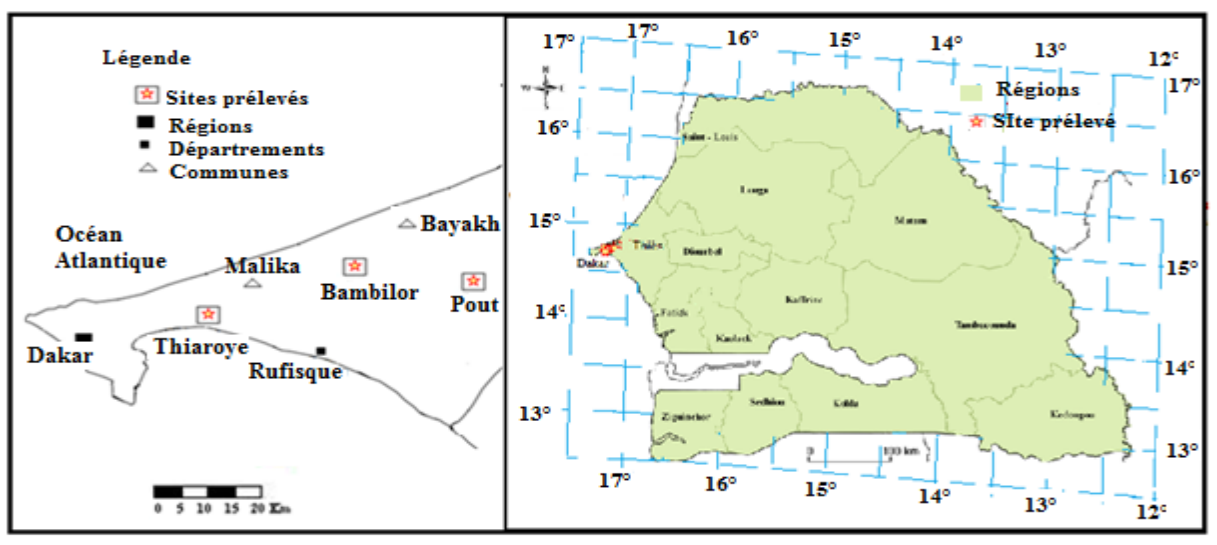

Figure 1 : Zone d'étude.

\section{Collecte des échantillons de bourses de Fabricius}

Des échantillons de bourses de Fabricius ont été collectés en 2012 et 2014 sur des sujets suspectés atteints de la maladie de Gumboro dans des élevages avicoles semiintensifs de la région de Dakar. Dans chaque élevage suspecté infecté, un échantillon de bourse de Fabricius a été prélevé sur un sujet malade pris au hasard. De plus, deux échantillons, datant de 2006, collectés dans le cadre d'un programme de recherche sur la maladie a été analysé. Les échantillons ont été d'abord soumis à un examen macroscopique puis placés dans des tubes de prélèvement stériles et transportés sous chaine de froid au Laboratoire National de l'Elevage et de Recherches Vétérinaires (LNERV) de Dakar pour la détection du virus. Par ailleurs, des informations relatives à la localisation de l'élevage, à la spéculation, à la vaccination, à l'âge des sujets et à l'effectif de la bande conduite ont été recueillies sur des fiches de renseignements.

\section{Détection du virus de la maladie de Gumboro \\ Préparation des échantillons de bourses de Fabricius}

Les échantillons sont hachés et transférés dans un broyeur de tissu. Après broyage, une suspension de $10 \%(\mathrm{w} / \mathrm{v})$ a été préparée dans du PBS $(\mathrm{pH} 7,2)$ renfermant de la pénicilline $(1000 \mathrm{IU} / \mathrm{ml})$ de la streptomycine $(100 \mu \mathrm{g} / \mathrm{ml})$ de la gentamycine (10 $\mu \mathrm{g} / \mathrm{ml})$ et du fungizone $(2,5 \mu \mathrm{g} / \mathrm{ml})$.
Chaque échantillon a été préparé en une suspension centrifugée à $12,000 \mathrm{rpm}$ à $4{ }^{\circ} \mathrm{C}$ pendant 30 minutes puis, le surnageant collecté pour être soumis à la l'extraction de l'ARN viral.

\section{Extraction de l'ARN viral et RT-PCR}

L'extraction de l'ARN viral a été conduite à l'aide d'un Mini Kit QIAamp RN, Qiagen, en suivant les recommandations du fabricant. Les ARNs extraits sont soumis à la RT-PCR qui a été conduite avec un Kit One Step RT-PCR Qiagen. En effet, 743 pb comprises entre les nucléotides 737 et 1479 de la région hypervariable du génome codant pour la protéine de structure VP2 ont été amplifiés en utilisant les amorces 743-1 (5'GCCCAGAGTCTACACCAT-3') et 743-2 (5'-CCCGGATTATGTCTTTGA-

(Jackwood et al., 2011). Ces amorces permettent d'amplifier cette région du génome du segment A de toutes les souches du sérotype $1 \mathrm{du}$ virus. Pour une réaction, un volume total de $50 \mu$ contenant $5 \mu \mathrm{l}$ de l'ARN total, $10 \mu \mathrm{l}$ de Buffer Tampon PCR $5 \mathrm{x}, 3 \mu \mathrm{l}$ $(10 \mu \mathrm{M})$ de chaque amorce, $2 \mu \mathrm{l}$ de $10 \mathrm{mM}$ de dNTPs mix, $2 \mu l$ de l'enzyme One Step RTPCR mix, $10 \mu \mathrm{l}$ de Q $5 X$ Solution et $15 \mu \mathrm{l}$ d'eau sans Rnase est préparé. Les tubes PCR sont placés dans le Thermocycleur (Bio-Rad MJ Mini Personal Thermal Cycler). Il a été programmé pour 35 cycles d'amplification, une RT incubation à $50{ }^{\circ} \mathrm{C}$ pendant $30 \mathrm{mn}$ suivie par une phase d'activation de la Taq polymérase enzyme à $95{ }^{\circ} \mathrm{C}$ pendant $15 \mathrm{mn}$. L'activation de l'enzyme est suivie de 35 cycles de $30 \mathrm{~s}$ à $95^{\circ} \mathrm{C}$, de $1,5 \mathrm{mn}$ à $53{ }^{\circ} \mathrm{C}$ et 
de $1 \mathrm{mn}$ à $72{ }^{\circ} \mathrm{C}$. A la fin des 35 cycles d'amplification, la réaction a été maintenue à $72{ }^{\circ} \mathrm{C}$ pendant $7 \mathrm{mn}$. Après amplification, les échantillons sont conservés entre 2 et $8{ }^{\circ} \mathrm{C}$ avant d'être analysés sur gel d'agarose.

\section{Contrôle des produits d'amplification sur gel d'agarose}

Les produits d'amplification sont séparés sur gel d'agarose $2 \%$ en tampon TAE (Tris-Acetate-EDTA) 1 x coloré au bromure d'éthidium. Pour chaque échantillon, $8 \mu \mathrm{du}$ produit d'amplification sont mélangés à $2 \mu \mathrm{l}$ d'une solution de charge $6 \mathrm{X}$ et déposés dans un puits du portoir plongé dans la cuve. Les échantillons sont comparés à un marqueur de masse moléculaire de $1000 \mathrm{pb}$ (Promega) déposé au premier puits du portoir. Après cette étape de remplissage des puits, l'appareil d'électrophorèse est mis en marche à 100 volts pour faire migrer le gel. Au bout de 30 $\mathrm{mn}$, le portoir est retiré de la cuve et le gel déposé sur le système de photographie pour visualiser par transillumination ultraviolette la bande du produit d'amplification.

\section{Séquençage des produits d'amplification \\ Dix échantillons de produits} d'amplification ont été envoyés en France, à l'Ecole Nationale Vétérinaire de Toulouse pour l'étape du séquençage. Les échantillons sont d'abord purifiés avec le Kit QIAquick PCR, Qiagen, en suivant les indications du fabricant. Le séquençage a été conduit avec un appareil 3130XL Applied Biosystems capillary sequencer du Plateau de Génomique GeT-Purpan, UDEAR UMR 5165 CNRS/UPS, CHU PURPAN, Toulouse, France. Les amorces utilisées pour amplifier l'ARN viral ont été réutilisées pour le séquençage.

\section{Analyse phylogénétique}

Le logiciel MEGA version 5.1 (Tamura et al., 2011) a été utilisé pour analyser les séquences nucléotidiques obtenues. Elles sont alignées et comparées à des séquences de référence isolées en Europe, en Asie, aux Etats Unis d'Amérique et en Afrique à l'aide du programme Clustal W inclus dans MEGA version 5.1. L'analyse phylogénétique portant sur la région du génome codant pour le « domaine variable de la protéine de structure VP2 » a été effectuée en utilisant la méthode neighbour-joining à 1000 répliques boostrap. La méthode Pairwise distance a été utilisée pour estimer la divergence entre les souches. Les isolats séquencés sont par la suite enregistrés sur la base de données de GenBank.

\section{RESULTATS}

\section{Détection du virus de la maladie de Gumboro}

Sur les 12 échantillons de bourses de Fabricius soumis à la détection du virus par la technique RT-PCR, 10 échantillons se sont révélés positifs et 02 négatifs. Les produits d'amplification sont visualisés par transillumination ultraviolette et des bandes de $743 \mathrm{pb}$ sont obtenues (Figure 2).

\section{Séquençage et analyse phylogénétique}

Sur les 10 échantillons purifiés et séquencés, 08 ont fourni des résultats exploitables, qu'on pouvait analyser. Sur ces 08 souches séquencées, 03 souches provenaient de la commune de Thiaroye, 03 de la commune de Bambilor et 02 de la commune de Pout. Les séquences d'acides nucléiques comprises entre les nucléotides 737 et 1479 de la région hypervariable du génome VP2 sont déterminées pour ces souches. Les séquences d'acides nucléiques et aminés sont enregistrées sur la base de données de GenBank et des numéros d'accès leur sont attribués (Tableau 1).

\section{Analyse des séquences nucléotidiques}

Les résultats sur l'analyse des séquences nucléotidiques des 08 souches isolées dans la région de Dakar (IBDV/DK2/SN/2014, IBDV/DK4/SN/2014, IBDV/DK5/SN/2014， IBDV/DK1/SN/2006, IBDV/DK4/SN/2012, IBDV/DK7/SN/2012, IBDV/DK6/SN/2014 et IBDV/DK7/SN/2014) sont comparés à des séquences de souches africaines, asiatiques, européennes et américaines. L'analyse a révélé une identité en acides nucléiques entre toutes les 08 souches sénégalaises comprise entre 97 et $100 \%$.

Les souches IBDV/DK2/SN/2014, IBDV/DK4/SN/2014, IBDV/DK5/SN/2014, IBDV/DK1/SN/2006, IBDV/DK4/SN/2012, 
IBDV/DK7/SN/2012, IBDV/DK6/SN/2014 et IBDV/DK7/SN/2014 ont une similarité entre elles en acides nucléiques comprise entre 99 et $100 \%$. La comparaison de ces souches aux souches nigérianes IBDV33/Abuja.NG/2011, IBDV63/Kaduna.NG/2009, IBDV43/Kaduna.NG/2011, IBDV79/Kaduna.NG/2011 et IBDV2/Kaduna.NG/2009 a révélé une identité en acides nucléiques supérieure à $99 \%$. Sur les 08 souches sénégalaises, seule la souche IBDV/DK1/SN/2006 est très proche des souches de référence OKYM et Cro-Ig/02 isolées respectivement en Asie et en Europe avec une identité en acide nucléique supérieure à $99 \%$.

\section{Analyse phylogénétique}

L'analyse phylogénétique portant sur la séquence nucléotidique de la région du génome codant pour le «domaine variable de la protéine de structure VP2 » a révélé que les 08 souches isolées dans la région de Dakar appartiennent au sérotype 1 et sont toutes classées dans le génotype des souches «hypervirulentes». Dans ce génotype, ces souches se répartissent dans 2 sous-génotypes distincts: VV2 et VV3. Le premier sousgénotype (VV2) est constitué par les souches IBDV/DK2/SN/2014, IBDV/DK4/SN/2014, IBDV/DK5/SN/2014, IBDV/DK4/SN/2012, IBDV/DK7/SN/2012, IBDV/DK6/SN/2014 et IBDV/DK7/SN/2014 et les souches nigérianes IBDV33/Abuja.NG/2011, IBDV63/Kaduna.NG/2009, IBDV43/Kaduna.NG/2011, IBDV79/Kaduna.NG/2011 IBDV2/Kaduna.NG/2009. Le deuxième sousgénotype (VV3) est constitué par la souche IBDV/DK1/SN/2006 et les souches de référence OKYM, Cro-Ig/02, 062Osun.NIE98 et IBDV80/Kaduna.NG/2011(Figure 3).

\section{Analyse des séquences d'acides aminés déduites}

L'analyse des séquences d'acides aminés a révélé une similarité de $100 \%$ entre les souches IBDV/DK2/SN/2014, IBDV/DK4/SN/2014, IBDV/DK5/SN/2014, IBDV/DK4/SN/2012, IBDV/DK7/SN/2012, IBDV/DK6/SN/2014 et IBDV/DK7/SN/2014, isolées dans la région de Dakar. La comparaison entre ces souches et les souches nigérianes (IBDV33/Abuja.NG/2011, IBDV63/Kaduna.NG/2009, IBDV43/Kaduna.NG/2011, IBDV79/Kaduna.NG/2011 et IBDV2/Kaduna.NG/2009) révèle aussi une similarité en acides aminés de $100 \%$.

Par contre, la similarité entre les souches IBDV/DK2/SN/2014, IBDV/DK4/SN/2014, IBDV/DK5/SN/2014, IBDV/DK4/SN/2012, IBDV/DK7/SN/2012, IBDV/DK6/SN/2014 et IBDV/DK7/SN/2014 et la souche IBDV/DK1/SN/2006 aussi isolée dans la région de Dakar est de $99 \%$. La séquence d'acide aminé de la souche IBDV/DK1/SN/2006 est à 100\% identique à la séquence d'acide aminé de la souche de référence OKYM isolée en 1991 au Japon. Les 08 souches isolées dans la région de Dakar citées ci-dessus présentent l'Alanine à la position 222, l'Isoleucine aux positions 256 et 294 et la Sérine à la position 299, évocatrices de souches «hypervirulentes». Seule la souche IBDV/DK1/SN/2006 présente à la position 300 l'Acide glutamique et les autres souches ont l'Alanine à la position 300 (Figure 4).

\begin{tabular}{|c|c|c|c|c|c|c|c|}
\hline Isolat & Date & Localité & Age & Vacc. & Production & $N^{\circ}$ GenBank & Génotype \\
\hline IBDV/DK $2 / \mathrm{SN} / 2014$ & $01 / 03 / 2014$ & Thiaroye & 24 jours & + & Chair & KP773429 & VV \\
\hline IBDV/DK $5 / \mathrm{SN} / 2014$ & $18 / 07 / 2014$ & Thiaroye & 17 jours & + & Chair & KP773431 & VV \\
\hline IBDV/DK4/SN/2012 & $17 / 11 / 2012$ & Thiaroye & 25 jours & + & Chair & KP773435 & VV \\
\hline IBDV/DK $7 / \mathrm{SN} / 2012$ & $23 / 09 / 2012$ & Bambilor & 39 jours & + & Poulette & KP773436 & VV \\
\hline IBDV/DK6/SN/2014 & $28 / 08 / 2014$ & Pout & 27 jours & + & Chair & KP 773437 & VV \\
\hline IBDV/DK $7 / S N / 2014$ & $13 / 07 / 2014$ & Bambilor & 36 jours & + & Poulette & KP773438 & VV \\
\hline
\end{tabular}

+ : Vaccination par voie orale avec soit un vaccin vivant à souche «intermédiaire » ou à souche « intermédiaire plus » Vacc. : Vaccination NI : Non informé ; VV : Very virulent ; CV : Classical virulent. 


\section{Légende :}

VV: Grocpe des souches « hypervirlentes si subdivisées en 3 sous groupes VVl, VV2 et VV3

VV1 : 050 Oyo.NIE/9S, AJ586956; 342 Dyo.NIE00, AJ5369:9; 034 Oyo.NIE99, AJ586953 et 032

Ogur.NIE 97, AJ 586934 ;

VV2 : IBDV33/Akuja.NG/2011, JX424059; IBDV61/Kaduna NG/2J09, JX424069; IBDV43/Kaduna NG/2011

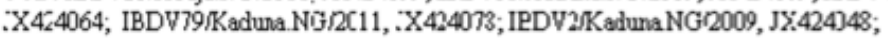

BDV 58Kaduna HG/2010, JX $<24067$;

VV3 : IBDV80/Kadune.NG/2011, JX424079.Cro-IgC2, EJ184685; OKYMM, D 49706 ;

CV: Groupe des sjuches «classiques 》: (Faragher/S2.70, Y14058; Zu-1/72t-1115, D00867; STC, D00499.

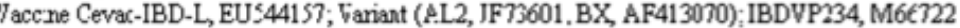

- Souches isolées an Sénégal
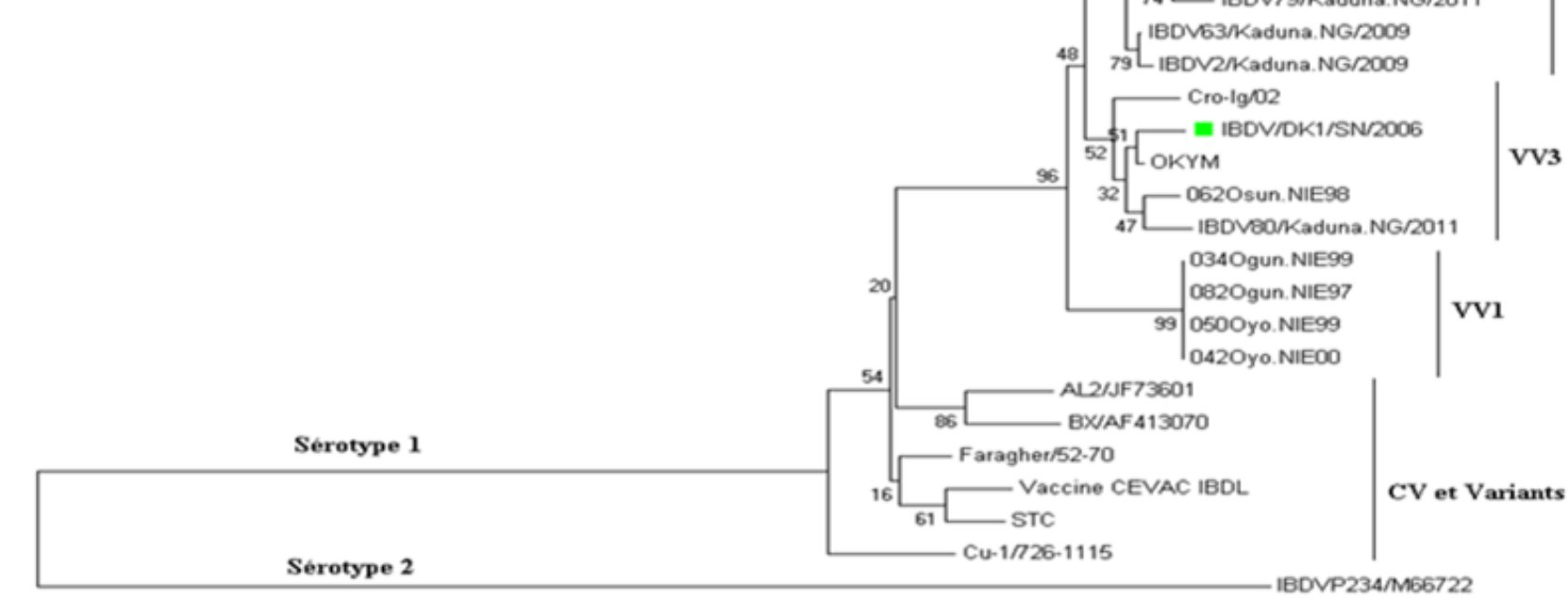

30 - 18 - IBDV43/Kaduna.NG/2011

IBDV63/Kaduna. NG/2009

$79 L_{180 V / \text { Kaduna.NG/2009 }}$ Cro-lg/02

- IBDV/OK1/SN/2006

16 95 . $1 \mathrm{l}=18 \mathrm{BV} / \mathrm{OK} \mathrm{K} / \mathrm{SN} / 2012$ IBDV/OKT/SN/2012

Figure 3 : Arbre phylogénétique des souches isolées à Dakar. 
A. Badji et al. / Int. J. Biol. Chem. Sci. 13(1): 196-206, 2019

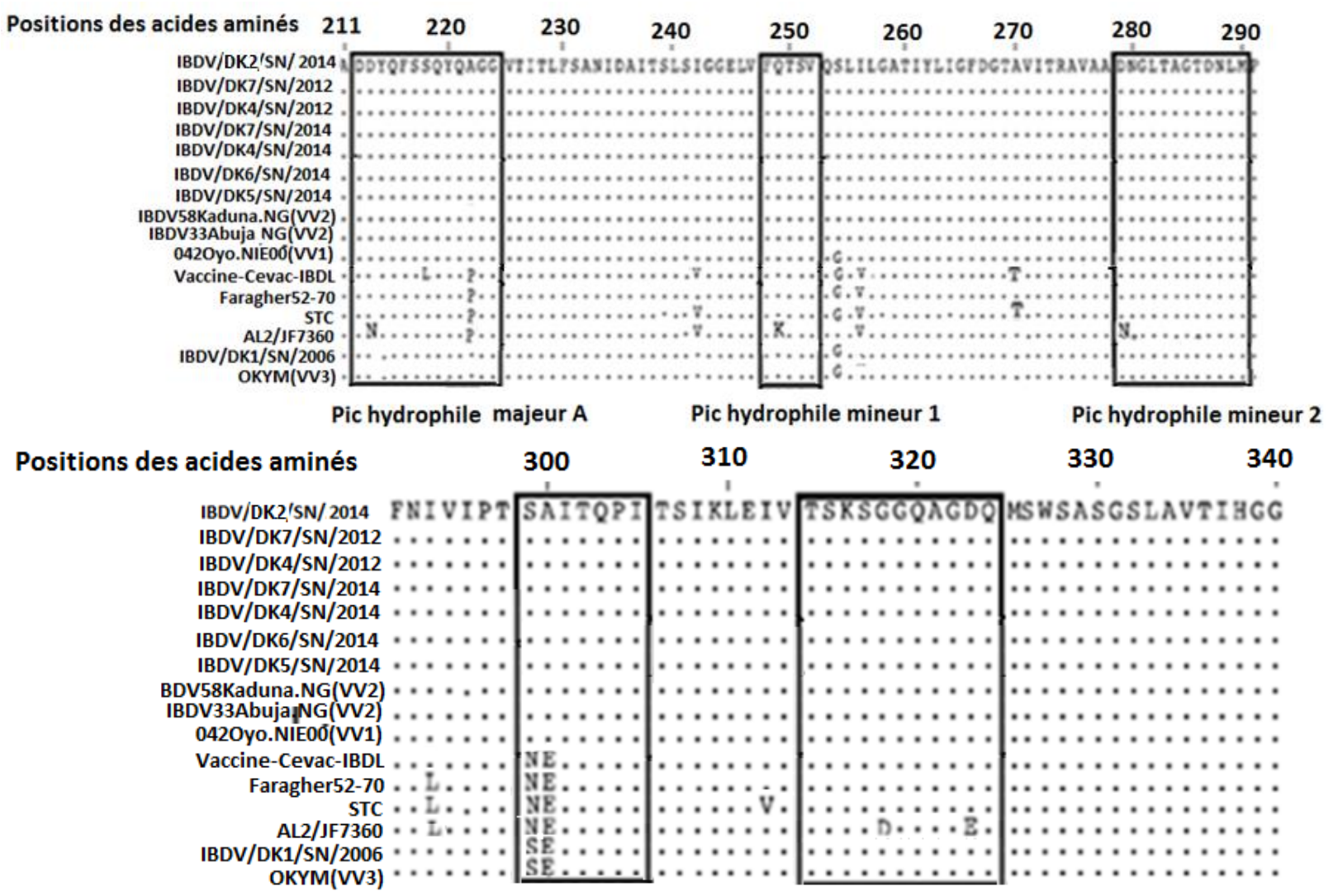

Figure 4: Séquences d'acides aminés du « domaine variable de la protéine de structure VP2 » comprises entre les acides aminés 211 et 340 des 08 souches isolées à Dakar comparées à des séquences d'acides aminés de souches « hypervirulentes » : OKYM (VV3), 042 Oyo.NIE.00 (VV1), IBDV58.Kaduna.NG/2010 (VV2), IBDV33Abuja.NG/2011 (VV2), de souches «Variantes »: AL2, JF73601, de souches « classiques »: STC, Faragher/52-70 et Vaccine Cevac-IBD-L. 


\section{DISCUSSION}

Dans la présente étude, le virus a été détecté par amplification de $743 \mathrm{pb}$ de la région du génome codant pour la protéine structurale VP2 par la technique RT-PCR. Sur les prélèvements positifs, le produit d'amplification était visible sur la photo du gel d'électrophorèse sous forme d'une bande horizontale observée à la hauteur des 743 pb $\mathrm{du}$ marqueur d'échelle. De pareilles observations ont été rapportées par Jackwood et al. (2011) avec les mêmes amorces d'amplification.

L'analyse des séquences d'acide nucléique et l'analyse phylogénétique ont révélé que les huit souches identifiées appartiennent au sérotype 1 qui regroupe les souches pathogènes du virus, témoignant la présence de la maladie dans les exploitations avicoles semi-intensives de Dakar. De plus, toutes les huit souches appartiennent au génotype des souches «hypervirulentes ». Ce génotype est subdivisé en trois sousgénotypes: VV1, VV2 et VV3 et sur les huit souches de ce génotype, sept souches sont classées dans le sous-génotype VV2 avec les souches nigérianes IBDV33/Abuja.NG/2011, IBDV63/Kaduna.NG/2009,

IBDV43/Kaduna.NG/2011,

IBDV79/Kaduna.NG/2011

et

IBDV2/Kaduna.NG/2009, une souche dans le sous-génotype VV3 avec la souche de référence OKYM. Selon une étude portant sur le « domaine variable de la protéine de structure VP2 ", on s'attend à ce que, dans le monde entier, environ 60 à $76 \%$ des isolats du virus de la maladie de Gumboro appartiennent au génotype des souches «hypervirulentes » et le reste, au génotype des souches « classiques» et « variantes » (Jackwood et Sommer-Wagner, 2007 ; He et al., 2012). Les souches isolées à Dakar sont très proches des souches nigérianes classées dans le sousgénotype VV2. Cette forte similarité en acide nucléique serait due à la résistance du virus à de nombreux désinfectants qui favorisent la circulation des souches dans la sous-région Ouest africaine à travers les transactions commerciales. La circulation de la souche IBDV/DK1/SN/2006 trouverait son explication dans les transactions commerciales entre le Sénégal et les pays d'Europe, d'Amériques et d'Asie. En effet, les importations de viande de volaille ont connu une évolution croissante de 2000 à 2004. Parallèlement à ces importations de viande, les œufs à couver sont approvisionnés principalement par la France, la Belgique, l'Italie et le Maroc malgré la mise en place progressive d'élevages de reproducteurs par la Sénégalaise de Distribution de Matériels Avicoles au début des années 2000. Les importations de poussins d'un jour et des autres produits avicoles ne sont interdites qu'en 2005 suite à l'avènement de la grippe aviaire. Et tous ces produits avicoles importés, infectés peuvent constituer de véritables sources de propagation des souches virales d'un pays à l'autre. Par ailleurs, la circulation de souches de cette lignée est aussi rapportée en Tanzanie par Kasanga et al. (2007) et en Zambie par Kasanga et al. (2012).

L'analyse des séquences d'acides aminés déduites a révélé que les souches isolées à Dakar, sauf la souche IBDV/DK1/SN/2006 ont une similarité en acides aminés de $100 \%$ sur la région du génome codant pour le «domaine variable de la protéine de structure VP2». Ces résultats obtenus indiquent une stabilité antigénique sur ces souches «hypervirulentes». Semblables résultats ont été rapportés sur des souches du même génotype isolées en Tanzanie (Kasanga et al., 2007) au Nigéria (Zierenberg et al,. 2000) et en Italie (Martin et al., 2007). Toutefois, en Chine, dans les principales zones de production avicole, des souches « hypervirulentes » classées dans des groupes phylogénétiques très divergentes suite au séquençage de la région du génome codant 
pour le domaine variable de la protéine VP2, ont été signalées par $\mathrm{He}$ et al. (2012). Cet observation indique une probable évolution antigénique des souches «hypervirulentes » aboutissant à une émergence de «variants » dans le futur.

L'analyse des séquences d'acides aminés déduites a révélé que toutes les souches étudiées, classés dans le génotype des souches « hypervirulentes » portent le groupe d'acides aminés qui caractérisent ce génotype. Ces acides aminés sont : l'alanine à la position 222 (A), l'isoleucine aux positions 256 (I) et 294 (I) et la sérine à la position 299 (S) qui ont été rapportés dans plusieurs études (Hoque et al., 2001 ; Banda et al., 2004). Les souches isolées au Sénégal ont la glutamine à la position $253(\mathrm{Q})$, l'acide aspartique à la position 279 (D) et l'alanine à la position 284 (A). De pareils résultats ont été rapportés sur des souches «hypervirulentes» isolées en Tanzanie (Kasanga et al., 2007), en Zambie (Kasanga et al., 2012) au Nigéria (Adamu et al., 2013) et en Egypte (Sara et al., 2014).Toutes ces observations confirment la circulation d'une lignée proprement africaine, la lignée VV2.

\section{Conclusion}

La technique RT-PCR associée au séquençage a permis d'identifier sur le plan moléculaire les souches du virus isolées en 2006, 2012 et 2014 dans des élevages avicoles semi-intensifs de la région de Dakar. Par la même occasion, les liens de parentés génétiques entre les souches isolées à Dakar et les souches étrangères sont évalués. Ainsi, il a été observé que la majeure partie des souches du virus qui circulent à Dakar appartiennent au génotype des souches «hypervirulentes». Les souches sont très proches des souches nigérianes du même sous-génotype et constituent avec les souches tanzaniennes, zambiennes et éthiopiennes la lignée africaine VV2. Cette lignée est indépendante de la lignée européenne et asiatique VV3 où une seule souche sénégalaise y est classée sur les huit souches identifiées. Toutefois, l'origine des souches reste à clarifier et des investigations élargies dans la sous-région portant sur le segment B du génome, apporteraient plus de précisions.

\section{CONFLIT D'INTERETS}

Les auteurs déclarent qu'il n'existe aucun conflit d'intérêts concernant la publication de cet article.

\section{CONTRIBUTIONS DES AUTEURS}

La thématique de recherche a été mise en forme par AB. RBA et MML ont suivi et supervisé le travail de recherche. Ils sont aussi impliqués dans la correction et la mise en forme du manuscrit.

\section{REMERCIEMENTS}

Les auteurs remercient le personnel de la section virologie et de la section pathologies aviaires du LNERV de l'Institut Sénégalais de Recherches Agronomiques (ISRA) de Dakar, les Aviculteurs de la région de Dakar, les Docteurs vétérinaires du privé et du public, les Ingénieurs des travaux d'élevage, les PER de l'ISFAR/UT et les Agents Techniques d'Elevage, d'avoir considérablement participé dans la réalisation de cette étude.

\section{REFERENCES}

Adamu J, Owoade AA, Abdu PA, Kazeem HM, Fatihu MY. 2013. Characterization of field and vaccine infectious bursal disease viruses from Nigeria revealing possible virulence and regional markers in the VP2 minor hydrophilic peaks. Avian Pathology, 42(5): 420-433. DOI: 10.1080/03079457.2013.822055.

Banda A, Villegas P. 2004. Genetic characterization of very virulent infectious bursal disease viruses from Latin America. Avian Diseases, 48(3): 540-549. DOI: 10.1637/7157-12304R. 
Badji A, Diallo AA, Ducatez M, Tall F, Diop M, Mbengue, Lo MM, Thiongane Y, Guerin JL, Alambedji RB. 2016. Genetic evolution of infectious bursal disease virus in Senegal. Journal of Veterinary Medicine and Animal Health, 8(12): 237243. DOI: $10.5897 / J V M A H 2016.0521$.

Badji A, Lo MM, Alambedji RB. 2017. Facteurs de risque liés à l'apparition de la maladie de Gumboro en aviculture semiintensive au Sénégal. International Journal of Biological and Chemical Sciences, 11(4): 1515-1558. DOI: http://dx.doi.org/10.4314/ijbcs.v11i4.182.

Boko MA, Dougnon TV, Bankolé HS, Dougnon TJ, Ahouangninou C, Cledjo P, Soumanou M. 2015. Pratiques d'élevage avicole au Sud-Bénin (Afrique de l'Ouest) et impacts sur l'hygiène des fumiers produits. International Journal of Biological and Chemical Sciences, 9(6): 2740-2753.

DOI: http://dx.doi.org/10.4314/ijbcs.v9i6.18.

Eterradossi N, Cardinal E, Arnauld C, Tekaia F, Toquin D, Le coq H, Rivallan G, Guittet M, Domenceh J, Van den berg TP, Skinner MA. 1999. Antigenic and genetic relationships between European very virulent infectious bursal disease viruses and an early West African isolate. Avian Pathology, 28: 36-46.

Hassan MK. 2004. Very virulent Infectious Bursal Disease Virus in Egypt: epidemiology, isolation and immunogenicity of classic vaccine. Veterinary Research, 28: 347-350.

He X, Wei P, Yang X, Guan D, Wang G, Qin A. 2012. Molecular epidemiology of infectious bursal disease viruses isolated from Southern China during the years 2000-2010. Virus Genes, 45(2): 246-255. DOI: https://doi.org/10.1007.

Hoque MM, Omar AR, Chong L K, Hair-Bejo M, Aini I. 2001. Pathogenicity of Ssp Ipositive infectious bursal disease virus and molecular characterization of the VP2 hypervariableregion. Avian Pathology, 30(4): $\quad 369-380 . \quad$ DOI: 10.1080/03079450120066377.

Jackwood DJ, Wagner SS. 2007.Genetic characteristics of infectious bursal disease viruses from four continents. Virology, 365(2): $\quad 369-375$. DOI: 10.1016/j.virol.2007.03.046.

Jackwood DJ, Sommer-Wagner SE, Crossley BM, Stoute ST, Woolcock PR, Charlton BR. 2011. Identification and pathogenicity of a natural reassortant between a very virulent serotype 1 infectious bursal disease virus (IBDV) and a serotype 2 IBDV. Virology, 420(2): 98-105. DOI: 10.1016/j.virol.2011.08.023.

Jembery S, Stacey EL, Fekadu K, Robert MC, Esayas G, Haileleul N, Kassahun Gelagay A. 2014. Genetic characterisation of infectious bursal disease virus isolates in Ethiopia. Acta Tropica, 130: 39-43.

Kasanga CJ, Yamaguchi T, Munang'andu HM, Wambura PN, Ohya K, Fukushi H. 2012. Genomic sequence of infectious bursal disease virus from Zambia suggests evidence for genome re-assortment in nature. Onderstepoort Journal of Veterinary Research, 79: E1. DOI: 10.4102/ojvr.v79i2.473.

Kasanga CJ, Yamaguchi T, Wambura P N, Maeda-Machang'u AD, Ohya K, Fukushi H. 2007. Molecular characterization of infectious bursal disease virus (IBDV): diversity of very virulent IBDV in Tanzania. Archives of Virology, 152(4): 783-790. DOI: 10.1007/s00705-006-08985.

Kasanga CJ, Yamaguchi T, Munang'andu HM, Ohya K, Fukushi H. 2013. Genomic sequence of an infectious bursal disease virus isolate from Zambia: classical attenuated segment $\mathrm{B}$ reassortment in nature with existing very virulent segment A. Archives of Virology, 158: 685-689. DOI: 10.1007/s00705-012-1531-4. 
Martin AM, Fallacara F, Barbieri I, Tosi G, Rivallan G, Eterradossi N, Ceruti R, Cordioli P. 2007. Genetic and antigenic characterization of infectious bursal disease viruses isolated in Italy during the period 2002-2005. Avian Diseases, 51 (4): 863-872. DOI: 10.1637/7904-020107REGR.1.

Mardassi H, Khabouchi N, Ghram A, Namouchi A, Karboul A. 2004. A Very Virulent Genotype of Infectious Bursal Disease Virus Predominantly Associated with Recurrent Infectious Bursal Disease Outbreaks in Tunisian Vaccinated Flocks. Avian Diseases, 48: 829-840.

Mac Lachlan NJ, Dubovi EJ. 2011. Fenner's Veterinary Virology. Elsevier: London; 293-297.

Owoade AA, Mulders MN, Kohnen J, Ammerlaan W, Muller CP. 2004. High sequence diversity in infectious bursal disease virus serotype 1 in poultry and turkey suggests West-African origin of very virulent strains. Archives of Virology, 149: 653-672.

Sara AM, Abdel SA, Hussein AH. 2014. Molecular genotyping of the infectious bursal disease virus (IBDV) isolated from Broiler Flocks in Egypt. International Journal of Veterinary Science Medicine, 2: 46-52.

Tamura K, Peterson D, Peterson N, Stecher G, Nei M, Kumar S. 2011. MEGA5: molecular evolutionary genetics analysis using maximum likelihood, evolutionary distance, and maximum parsimony methods. Molecular Biology and Evolution, 28(10): 2731-2739. DOI: 10.1093/molbev/msr121.

Traoré EH. 2006. Première évaluation de la structure et de l'importance du secteur avicole commercial et familial en Afrique de l'Ouest. Rapport du Sénégal; 52 p.

Zierenberg K, Nieper H, Van den Berg TP, Ezeokoli CD, Voss M, Muller H. 2000. The VP2 variable region of African and German isolates of infectious bursal disease virus: comparison with very virulent, "classical" virulent, and attenuated tissue culture-adapted strains'. Archives of Virology, 145: 113-125. DOI: 10.1007/s007050050009. 